\title{
Sur les interactions entre politiques de dette publique et de transfert
}

\author{
Marie Bessec, Banque de France, Université Paris-Dauphine (LEDa), \\ Audrey Desbonnet, Université Paris-Dauphine (LEDa), \\ Sumudu Kankanamge, Toulouse School of Economics (GREMAQ) et CEPREMAP, \\ Thomas Weitzenblum, Université Lille 2, EQUIPPE et CEPREMAP!
}

\begin{abstract}
Résumé
Dans cet article, nous analysons les interactions entre les politiques de dette publique et de transfert dans un modèle à la Bewley (1986)-Huggett (1993)-Aiyagari (1994). A l'instar de Desbonnet et Weitzenblum (2011), nous étendons l'approche de Floden (2001) en considérant explicitement la dynamique transitoire d'un état stationnaire à un autre, et étudions les interactions entre ces deux politiques. Dans un premier temps, nous montrons qu'il est possible de mettre en oeuvre une politique de désendettement de l'Etat, en l'associant à un ajustement transitoire du transfert, et ceci en partant de situations où la dette aurait atteint un niveau élevé, en raison de la tentation de dévier mise en exergue par Desbonnet et Weitzenblum (2011). Dans un second temps, nous proposons un équilibre intégrant les tentations de dévier en termes à la fois de dette et de transferts. Nous montrons que l'optimum de long terme sur la dette et les transferts proposé par Floden (2001) n'est pas stable au regard de ces déviations. Quantitativement, l'équilibre que nous obtenons s'éloigne considérablement de ce dernier.
\end{abstract}

\section{Introduction}

Si la brûlante actualité situe la dette publique au coeur du débat économique, les mécanismes macroéconomiques associés à celle-ci sont analysés depuis longtemps et

*Les vues exprimées dans cet article n'engagent que l'auteur et ne reflètent pas nécessairement celles de la Banque de France.

${ }^{\dagger}$ Correspondance. Adresse : EQUIPPE - Université Lille 2/Faculté de Droit, 1 place Déliot, 59000 Lille. Email : thomas.weitzenblum@univ-lille2.fr. Tel : +33 (0)3 20907535. 
ont donné lieu à une vaste littérature. La question de l'optimalité de la politique fiscale en général, et de la dynamique de la dette publique en particulier, a été traitée originellement par Barro (1974, 1979). Ce dernier montre que le lissage de l'imposition implique que le taux de taxe optimal suit une marche aléatoire. Le gouvernement devrait s'endetter pour lisser les effets distorsifs de l'imposition. Depuis, d'autres travaux se sont attachés à éprouver ce résultat en dotant l'approche de davantage de fondements microéconomiques (Lucas et Stokey (1983), Aiyagari et al. (2002)).

Récemment, les modèles à la Bewley (1986)-Huggett (1993)-Aiyagari (1994), qui intègrent le comportement d'épargne des ménages face à un risque de revenu non assurable, se sont emparés de cette question. Aiyagari et McGrattan (1998) déterminent le niveau optimal de dette publique fondé sur la comparaison d'états stationnaires. A l'optimum, la dette publique représente $2 / 3 \mathrm{du}$ PIB, qui se trouve être le niveau moyen observé aux Etats-Unis depuis 1945. La perte en bien-être à s'écarter de ce ratio optimal se trouve cependant faible : en l'absence de toute dette, le coût s'élève à seulement $0.08 \%$ de consommation. Floden (2001) soulève la question de la détermination conjointe de la dette publique et des transferts. En particulier, l'auteur montre que l'optimum est atteint pour un niveau de dette publique et de transferts égaux respectivement à $-100 \%$ et $23 \%$ du PIB. Par ailleurs, les gains de bien-être sont sensiblement plus élevés que lorsque l'Etat ajuste uniquement la dette publique. La redistribution en tant que telle a également donné lieu à d'autres évaluations dans le cadre de ces modèles (entre autres, Floden et Linde (2001)), qui se prêtent particulièrement bien à l'analyse du comportement des agents face au risque de revenu et aux inégalités.

Desbonnet et Weitzenblum (2011) montrent que les gains qui découlent de l'accroissement de la dette publique sont nettement plus élevés lorsque la transition d'un état stationnaire à un autre est explicitement modélisée. A la date où la dette publique s'élève, l'Etat dispose de ressources additionnelles lui permettant, par exemple, de réduire temporairement les impôts ou d'accroître les transferts. Cela se révèle bénéfique aux agents qui butent sur leur contrainte d'endettement, comme en témoignent les travaux de Daniel (1993) et Heathcote (2005). Plus précisément, Desbonnet et Weitzenblum (2011) montrent que les gains sont sept fois plus élevés que si la comparaison portait sur des états stationnaires. Par ailleurs, les auteurs illustrent la forte tentation de dévier vers davantage de dette publique en raison des effets de courte période.

Dans cette contribution, nous prolongeons le modèle de Desbonnet et Weitzenblum (2011) afin d'analyser les interactions potentielles entre les politiques de dette publique et de transferts. Puisque Desbonnet et Weitzenblum (2011) montrent que la 
tentation d'accroître la dette publique peut mener à des niveaux élevés de celle-ci, nous analysons dans quelle mesure des chocs conjoints de dette publique et de transferts peuvent remettre en cause ce résultat. Dans un premier temps, en partant d'une dette élevée, nous montrons que l'ajustement temporaire des transferts permet de rendre acceptable une politique de désendettement de l'Etat. Dans un second temps, nous envisageons la tentation de dévier, non pas seulement en termes de dette publique, mais également, et de manière symétrique, de transferts. Il apparaît que les tentations de dévier cessent pour un transfert de $16,5 \%$ du PIB, qui est un niveau intermédiaire entre le niveau de référence et l'optimum de long terme de Floden (2001). La dette publique demeure conséquente (495\% du PIB), quoique sensiblement inférieure à celle pour laquelle seules les tentations de dévier en termes de dette sont considérées.

Cet article est organisé comme suit. Dans la première partie, nous présentons brièvement le modèle de référence utilisé et définissons la dynamique transitoire de celui-ci. Dans une deuxième partie, nous présentons les différentes simulations de réduction de la dette publique puis de tentation de dévier. La troisième section est consacrée à la conclusion.

\section{Le modèle}

\subsection{Présentation du modèle}

Nous considérons le modèle de Floden (2001) lui-même repris par Desbonnet et Weitzenblum (2011). Il s'agit d'un modèle d'équilibre général avec marchés incomplets, risque de revenu et contrainte d'endettement. Le secteur de la production est modélisé par une firme représentative et des marchés de facteurs de production concurrentiels tels que :

$$
\begin{aligned}
& Y_{t}=F\left(K_{t}, Z_{t} N_{t}\right)=K_{t}^{\alpha}\left(Z_{t} N_{t}\right)^{1-\alpha} \\
& r_{t}+\delta=\frac{\partial Y_{t}}{\partial K_{t}} \text { et } w_{t}=\frac{\partial Y_{t}}{\partial N_{t}}
\end{aligned}
$$

avec $K$ le stock de capital agrégé et $N$ la quantité de main d'oeuvre agrégée. $Z$ est le progrès technique neutre au sens de Harrod, avec $Z_{t}=(1+g)^{t}$. Sur le sentier de croissance équilibrée, $w, Y$ et $K$ croissent au taux $g$ tandis que le taux d'intérêt $r$ demeure constant.

Le secteur des ménages est constitué d'un continuum d'agents à durée de vie infinie et de masse unitaire. Les ménages font face à un risque affectant leur produc- 
tivité. Ce risque idiosyncrasique est représenté par une chaîne de Markov décrivant les probabilités de transition entre les divers niveaux de productivité considérés. L'incomplétude des marchés et la contrainte d'endettement conduisent les ménages à lisser leur consommation en accumulant un actif financier unique. La contrainte budgétaire du ménage s'écrit :

$$
a_{t+1}=a_{t}+\left(r_{t} a_{t}+e_{t} w_{t} l_{t}\right)\left(1-\tau_{t}\right)+\operatorname{Tr}_{t}-c_{t}
$$

avec $c$ la consommation, $a$ le niveau de l'actif accumulé, e un choc de productivité idiosyncrasique sur le marché du travail, $l$ l'offre de travail, $T r$ un transfert forfaitaire mis en place par l'Etat et $\tau$ un taux de taxe proportionnel. L'utilité intertemporelle des agents est :

$$
U_{0}=E \sum_{t=0}^{\infty} \beta^{t} u\left(c_{t}, l_{t}\right)
$$

avec

$$
u\left(c_{t}, l_{t}\right)=\frac{c_{t}^{1-\rho} \exp \left[-(1-\rho) \zeta l_{t}^{1+\eta}\right]}{1-\rho}
$$

Nous reproduisons ci-dessous le programme du ménage une fois le modèle déflaté ${ }^{1}$ :

$$
\widehat{V}_{t}\left(\widehat{a}_{t}, e_{t}\right)=\underset{\widehat{c}_{t}, \widehat{a}_{t+1}, l_{t}}{\operatorname{Max}}\left\{u\left(\widehat{c}_{t}, l_{t}\right)+\beta(1+g)^{(1-\rho)} E_{t}\left[\widehat{V}_{t+1}\left(\widehat{a}_{t+1}, e_{t+1}\right) /\left(\widehat{a}_{t}, e_{t}\right)\right]\right\}
$$

sous la contrainte :

$$
\left\{\begin{array}{l}
\widehat{c}_{t}+\widehat{a}_{t+1}(1+g)=\widehat{a}_{t}+\left(r_{t} \widehat{a}_{t}+e_{t} \widehat{w}_{t} l_{t}\right)\left(1-\tau_{t}\right)+\widehat{T r}_{t} \\
\widehat{a}_{t+1} \geqslant 0, \widehat{c}_{t} \geqslant 0
\end{array}\right.
$$

L'Etat finance ses dépenses publiques $G_{t}$, les transferts $\operatorname{Tr}_{t}$ et les intérêts de la dette contractée par le passé à l'aide des impôts $T_{t}$ et de la dette $B_{t+1}$ nouvellement émise. La contrainte budgétaire de l'Etat une fois déflatée est la suivante :

$$
\widehat{G}_{t}+\widehat{T}_{t}+r_{t} \widehat{B}_{t}=(1+g) \widehat{B}_{t+1}-\widehat{B}_{t}+\widehat{T}_{t}
$$

Les instruments de politique économique retenus par le gouvernement sont $\gamma_{t}=$ $\widehat{G}_{t} / \widehat{Y}_{t}$ le ratio dépenses publique sur PIB, $\chi_{t}=\widehat{T} \widehat{r}_{t} / \widehat{Y}_{t}$ le ratio transfert sur PIB ainsi que $b_{t}=\frac{\widehat{B}_{t}}{\widehat{Y}_{t}}$ le ratio dette publique sur PIB.

1. Nous noterons une variable déflatée à l'aide d'un chapeau tel que $\widehat{x}_{t}=\frac{x_{t}}{(1+g)^{t}}$. 


\subsection{Définition de l'équilibre}

Dans cette section, nous définissons conjointement l'équilibre stationnaire et la dynamique transitoire du modèle.

L'équilibre stationnaire correspond à un équilibre où les variables stationnarisées sont constantes au cours du temps. Etant donné un jeu de paramètres de politique économique $\{\gamma, \chi, b\}$, l'équilibre stationnaire est défini par le vecteur:

$$
\left\{\widehat{c}(\widehat{a}, e), \widehat{a}^{\prime}(\widehat{a}, e), l(\widehat{a}, e), \Lambda(\widehat{a}, e), \widehat{K}, N, \widehat{Y}, r, \widehat{w}, \tau\right\}
$$

où $\Lambda(\widehat{a}, e)$ est la distribution des agents sur l'espace d'état, tel que :

1. Etant donnés $r, \widehat{w}, \tau$, et $\widehat{T r}=\chi \widehat{Y}$, les règles de décision $\left\{\widehat{c}(\widehat{a}, e), \widehat{a}^{\prime}(\widehat{a}, e), l(\widehat{a}, e)\right\}$ sont les solutions du programme (2),

2. $\Lambda(\widehat{a}, e)$ est l'unique distribution compatible avec les règles de décision décrites précédemment,

3. Les marchés du travail et du capital sont à l'équilibre :

$$
\begin{aligned}
& \widehat{K}+\widehat{B}=\sum_{e \in E} \int \widehat{a} \Lambda(\widehat{a}, e) d \widehat{a} \\
& N=\sum_{e \in E} \int e l(\widehat{a}, e) \Lambda(\widehat{a}, e) d \widehat{a}
\end{aligned}
$$

4. Les prix des facteurs sont tels que :

$$
\begin{aligned}
r & =F_{\widehat{K}}^{\prime}(\widehat{K}, N)-\delta \\
\widehat{w} & =\frac{F_{N}^{\prime}(K, Z N)}{Z}=F_{N}^{\prime}(\widehat{K}, N)
\end{aligned}
$$

5. La contrainte budgétaire de l'Etat est respectée :

$$
(r-g) \widehat{B}=\widehat{T}-(\widehat{G}+\widehat{T} r) \Longleftrightarrow(r-g) b=\tau\left(1-\delta \frac{\widehat{K}}{\widehat{Y}}+r b\right)-(\gamma+\chi)
$$

La dynamique transitoire du modèle permet de calculer le passage d'un état stationnaire vers un autre. Une définition théorique de cette dynamique est nécessaire. A cette fin, nous devons exprimer des conditions initiales décrivant l'état de tous les agents hétérogènes et du gouvernement à la date $t=0$. Etant données ces conditions initiales, la transition définit des sentiers pour les variables $r_{t}, \widehat{w}_{t}, \widehat{Y}_{t}, N_{t}, \widehat{K}_{t}, \widehat{B}_{t}, \widehat{T r}_{t}$, 
et $\tau_{t}$ qui sont cohérents avec les règles de décision des agents. Cependant, toutes les variables ci-dessous ne sont pas mutuellement indépendantes et la dynamique transitoire peut être résumée en suivant la trajectoire des variables $\left(r_{t}\right)_{t \geqslant 0},\left(N_{t}\right)_{t \geqslant 0}$. Nous pouvons à présent définir la dynamique transitoire elle-même :

Etant données les conditions initiales $\left\{\Lambda_{0}(.,),. \widehat{B}_{0}\right\}$ et le vecteur définissant la trajectoire des variables de politique économique $\left\{b_{t}, \chi_{t}, \gamma_{t}\right\}$, un équilibre dynamique à anticipations rationnelles est défini par :

$$
\left\{\widehat{c}_{t}(\widehat{a}, e), \widehat{a}_{t+1}(\widehat{a}, e), l_{t}(\widehat{a}, e), \Lambda_{t}(\widehat{a}, e), r_{t}, \widehat{w}_{t}, \tau_{t}, \widehat{Y}_{t}\right\}
$$

tel que :

1. A toute date $T$, étant donnés les vecteurs $\left\{\left(r_{t}\right)_{t \geqslant T},\left(\widehat{w}_{t}\right)_{t \geqslant T},\left(\tau_{t}\right)_{t \geqslant T},\left(\widehat{T r}_{t}=\chi_{t} \widehat{Y}_{t}\right)_{t \geqslant T}\right\}$, $\widehat{c}_{T}(\widehat{a}, e), \widehat{a}_{T+1}(\widehat{a}, e), l_{T}(\widehat{a}, e)$ sont les règles de décisions dérivées du programme (2),

2. A toute date $T, \Lambda_{T+1}$ s'obtient à partir de $\Lambda_{T}$ et des règles de décision ci-dessus,

3. A toute date $T$, les marchés du travail et du capital sont à l'équilibre :

$$
\begin{aligned}
N_{T} & =\sum_{e \in E} \int e l_{T}(\widehat{a}, e) \Lambda_{T}(\widehat{a}, e) d \widehat{a} \\
\widehat{K}_{T}+\widehat{B}_{T} & =\sum_{e \in E} \int \widehat{a} \Lambda_{T}(\widehat{a}, e) d \widehat{a}
\end{aligned}
$$

4. A toute date $T$, les prix des facteurs de production sont tels que :

$$
\begin{aligned}
r_{T} & =F_{\widehat{K}}^{\prime}\left(\widehat{K}_{T}, N_{T}\right)-\delta \\
\widehat{w}_{T} & =F_{N}^{\prime}\left(\widehat{K}_{T}, N_{T}\right)
\end{aligned}
$$

5. A toute date $T$, la loi d'évolution du stock de dette publique est la suivante :

$$
\left(\gamma_{T}+\chi_{T}\right) \widehat{Y}_{T}+r_{T} \widehat{B}_{T}=(1+g) \widehat{B}_{T+1}-\widehat{B}_{T}+\tau_{T}\left(\widehat{Y}_{T}-\delta \widehat{K}_{T}+r_{T} \widehat{B}_{T}\right)
$$

\subsection{Calibration}

La calibration du modèle reprend celle de Floden (2001) qui a également été employée par Desbonnet et Weitzenblum (2011). Nous nous limitons donc volontairement à une description sommaire de cette étape. La période du modèle est l'année. 
La productivité des agents a deux composantes. La composante permanente de la productivité peut prendre deux niveaux : haute ou basse. Pour chaque classe de productivité permanente, il existe une composante transitoire engendrée par le processus $A R(1)$ suivant :

$$
\log \left(q_{t}\right)=\psi \log \left(q_{t-1}\right)+\varepsilon_{t}
$$

avec $\psi$ la persistance du processus et $\varepsilon$ l'innovation avec $\varepsilon \sim N\left(0, \sigma_{\varepsilon}\right)$. Ce processus est transformé en une chaîne de Markov à 7 états en utilisant la procédure décrite dans Tauchen (1986). Au final, il existe 14 niveaux de productivité, divisés en deux sous-catégories: $E_{1}=\left\{e^{1}, e^{2}, e^{3}, e^{4}, e^{5}, e^{6}, e^{7}\right\}$ et $E_{2}=\left\{e^{8}, e^{9}, e^{10}, e^{11}, e^{12}, e^{13}, e^{14}\right\}$. La moitié des agents est dans $E_{1}$ tandis que le reste est dans $E_{2}$.

La calibration cible une valeur du ratio capital sur PIB de 2,5, en ajustant le facteur d'escompte $\beta$. Les valeurs des paramètres sont reportées dans le Tableau 1.

\begin{tabular}{ccccccccccc}
\hline \hline$\rho$ & $\beta$ & $\delta$ & $\gamma$ & $\alpha$ & $\eta$ & $\chi$ & $g$ & $\zeta$ & $\psi$ & $\sigma_{\varepsilon}$ \\
\hline 1,5 & 0,9885 & 0,075 & 0,217 & 0,3 & 2 & 0,082 & 0,0185 & 9,1449 & 0,9 & 0,21 \\
\hline \hline
\end{tabular}

TABLE 1 - Valeurs des paramètres du modèle

\section{Résultats}

Les simulations que nous opérons dans cette section ont pour objet d'étudier dans quelle mesure les politiques d'ajustement de la dette publique et des transferts interagissent entre elles. Dans un premier temps, nous illustrons l'effet de l'accompagnement du désendettement de l'Etat par une modification temporaire des transferts. Dans un second temps, nous étendons l'exercice de Desbonnet et Weitzenblum (2011), portant sur la tentation de dévier en termes de dette publique, à la double tentation de dévier en dette et en transferts.

\subsection{Quelles réductions de la dette publique sont-elles acceptables?}

Nous envisageons ici des politiques de réduction de la dette publique pour un niveau de transfert fixé, en décrivant l'intégralité de la dynamique d'ajustement d'un équilibre stationnaire à un autre. Les résultats de Floden (2001) illustrent les gains en bien-être de long terme provenant de la fixation de la dette à son niveau optimal. $\mathrm{Si}$ ces gains s'avèrent relativement faibles, Desbonnet et Weitzenblum (2011) ont montré 
que (i) la tentation de dévier vers davantage de dette, qui ne peut être appréhendée que sur la transition, est susceptible de mener vers des niveaux de dette nettement supérieurs à l'optimum de long terme et (ii) les gains de court terme sont sensiblement supérieurs à ceux tirés de comparaisons d'états stationnaires. Ces gains ne sont cependant pas uniformément répartis. En effet, les agents différant à la fois par des écarts permanents et temporaires de salaire, ainsi que par leur richesse financière courante, les effets de la hausse de la dette sont ressentis différemment suivant la situation individuelle de l'agent considéré. L'accroissement de la dette publique permet, à la date de son implémentation, de réduire temporairement le taux de taxe, ce qui est bénéfique aux agents dont les revenus sont élevés : soit parce qu'ils disposent d'un salaire temporairement haut, soit parce qu'ils disposent d'un stock d'actifs financiers conséquent. Cependant, une autre catégorie d'agents bénéficie relativement plus de cette politique : ceux qui sont contraints par la liquidité, et pour lesquels le relâchement, même temporaire, de cette contrainte, profite relativement plus qu'aux autres agents. Enfin, ce sont les classes intermédiaires en termes de revenu qui ne profitent que modérément de l'abaissement temporaire des prélèvements, et pour lesquels les effets néfastes à long terme d'une dette accrue -éviction du capital privé et hausse des prélèvements qui accroît l'effet distorsif des taxes- dominent.

Nous retenons le critère utilitariste comme mesure du bien-être social. Le critère utilitariste, soit la somme des utilités intertemporelles, s'écrit :

$$
W=\sum_{e \in E} \int_{\widehat{a} \in A} \widehat{V}(\widehat{a}, e) \Lambda(\widehat{a}, e) d \widehat{a}
$$

Les variations de bien-être sont évaluées à la date $t=0$, au moment de la mise en place de la politique économique, et sont calculées par rapport au bien-être à la même date en l'absence de pareil choc. Pour faciliter l'interprétation des résultats, ces variations d'utilité intertemporelle sont transformées en points de consommation permanente ${ }^{2}$.

Dans un premier temps, en partant de la situation de référence caractérisée par un ratio dette/PIB de $2 / 3$ et un transfert de $8,2 \%$, nous envisageons des accroissements définitifs et instantanés, à la date $t=0$, de la dette publique, d'un incrément de 1 point de PIB. Nous faisons l'hypothèse que la dette publique atteint, dès la première date de la transition $(t=0)$, son niveau final après le choc d'augmentation de la dette.

2. Pour chaque choc de politique économique, le critère utilitariste correspondant est transformé en niveau de consommation constante d'un agent à durée de vie infinie, dont la fonction d'utilité est donnée par (1). 
Ce niveau correspond au ratio dette/PIB de l'état final évalué avec le PIB de l'état final. Ainsi, sur le sentier de transition, la fraction effective que la dette représente, en proportion du PIB, varie, car le PIB lui-même varie, alors que la dette atteint instantanément son niveau final ${ }^{3}$. Si le choc élève le critère utilitariste, nous considérons que cette politique est souhaitée. Nous partons alors du nouvel équilibre stationnaire initial caractérisé par le ratio dette/PIB augmenté d'un point, et évaluons l'effet d'une augmentation supplémentaire d'un point, et ainsi de suite, tant que la hausse de 1 point de la dette élève le bien-être social. Les simulations révèlent que ces chocs engendrent une élévation du bien-être mesuré à la date initiale de la transition, jusqu'à ce que le ratio atteignent le niveau de $686 \%{ }^{4}$. Cette tentation de dévier vers davantage de dette est d'abord due aux gains, à court terme, d'une dette accrue, donc à une baisse du taux de taxe. En revanche, à long terme, le bien-être collectif est abaissé, en raison des effets distorsifs de la dette provenant de l'élévation du taux de prélèvement.

A la lumière de ce résultat, il est donc légitime de considérer une situation initiale où l'Etat aurait précédemment profité de ces gains temporaires en cédant à la tentation de courte période d'accroître la dette publique. En raison d'un argument de symétrie évident, on voit mal comment une réduction de la dette pourrait être souhaitable, lorsqu'une hausse l'était déjà. C'est pourquoi, nous posons à présent la question de savoir à quel autre choc fiscal la baisse de la dette publique pourrait être associée, de façon à rendre pareil ajustement souhaitable au regard du critère utilitariste ${ }^{5}$. Il est clair qu'au sein des multiples combinaisons de chocs fiscaux envisageables, nous nous pencherons uniquement sur un cas particulier, sans justifier pourquoi cette combinaison devrait être préférée à d'autres. L'objectif est avant tout ici de proposer des politiques économiques telles que le coût temporaire à réduire la dette puisse être surmonté, du moins au regard du critère utilitariste.

Parmi les choix possibles de chocs associés à celui de la baisse de la dette publique, celui du transfert forfaitaire apparaît naturellement. En effet, les effets à court terme de la dette mentionnés précédemment, et qui expliquent cette forte tentation de dévier

3. Ce choix de modélisation a été opéré, car, en son absence, les fluctuations du PIB, qui, en raison de celles de l'offre de travail et de l'accumulation de capital, peuvent être conséquentes, auraient un fort effet sur l'évolution de la dette sur la transition, et donc sur le taux de prélèvement.

4. Ce résultat diffère de celui de Desbonnet et Weitzenblum (2011), car les chocs opérés ici sont d'une amplitude de 1 point, et parce que la dette atteint instantanément son niveau final.

5. Précisons que les considérations liées au processus du choix collectif-aux types de choix alternatifs proposés aux agents- ne seront pas abordées ici. En d'autres termes, il est possible qu'une procédure de choix collectif soigneusement modélisée ne permettrait peut-être pas d'aboutir aux ajustements proposés. 

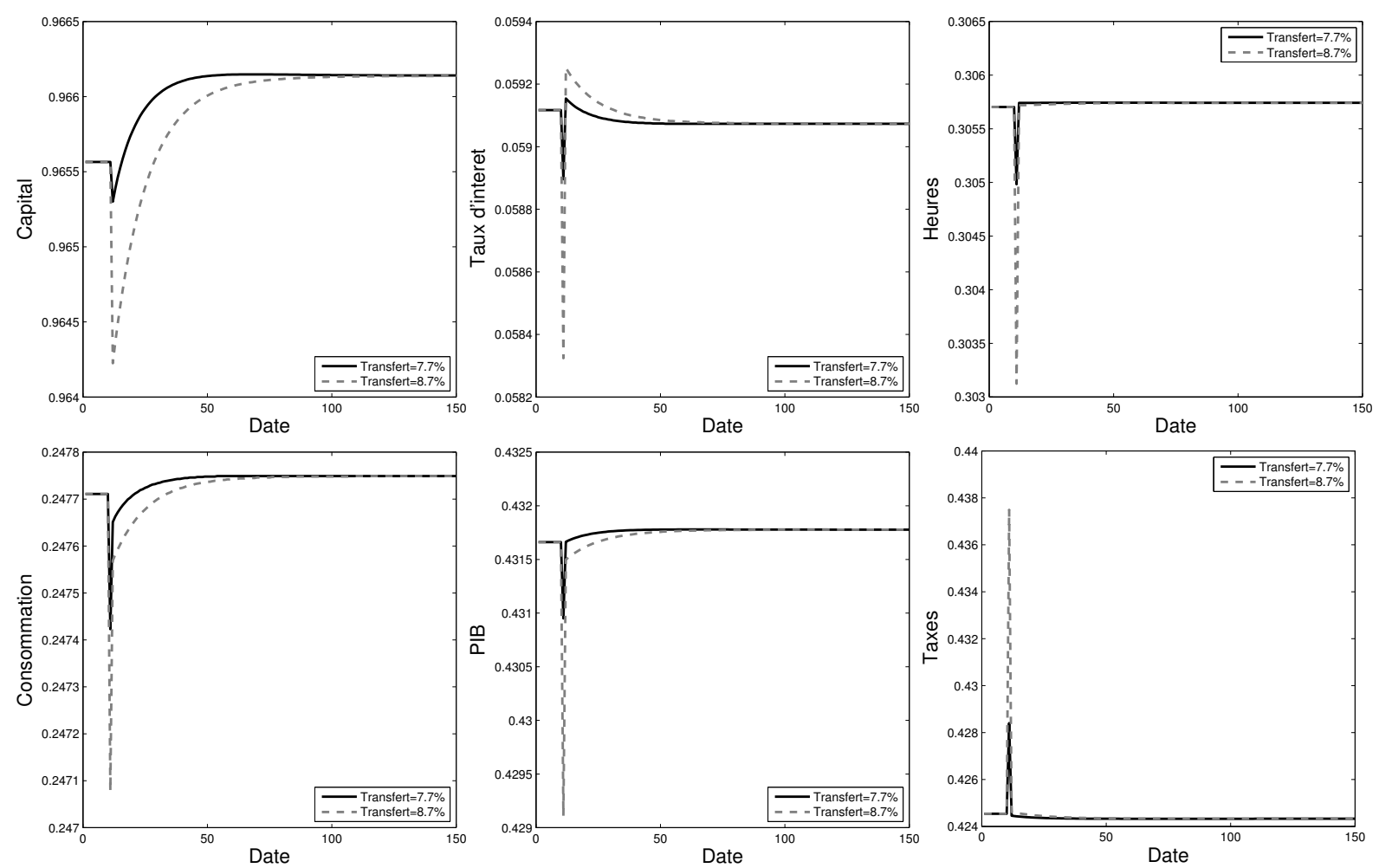

FIGURE 1 - Trajectoire du capital, du taux d'intérêt, de l'offre de travail, de la consommation, du PIB et des taxes pour une politique de baisse de la dette de $350 \%$ vers 349\%.

vers des niveaux élevés de dette, sont dus à la baisse du taux de taxe pour les agents disposant de revenus élevés, et au relâchement de la contrainte budgétaire, pour les agents butant sur la contrainte d'endettement. Dès lors, les premiers risquent de plaider en faveur d'une baisse des transferts, de manière à limiter l'ampleur de la hausse des prélèvements consécutive à un désendettement de l'Etat, lorsque les seconds devraient souhaiter une hausse des transferts. En d'autres termes, si le signe de la variation des transferts susceptible d'accompagner avantageusement le désendettement de l'Etat n'est pas évident a priori, il apparaît clairement que cette politique allègera en partie le coût du désendettement pour une fraction ou une autre de la population. Enfin, la baisse de la dette élevant, uniquement à la date de son implémentation, le taux de taxe, nous considérons ici un ajustement transitoire des transferts opéré lui aussi exclusivement à la date initiale. L'ajustement de la dette reste quant à lui permanent. De cette façon, l'équilibre final de long terme sera inchangé en termes de transferts, et correspondra à un niveau de dette inférieur.

En partant du ratio dette/PIB de 686\%, nous simulons la dynamique d'ajustement due à une réduction de ce ratio d'un point, en la combinant à une variation transi- 
toire des transferts, de signe et d'amplitude variables. Tant que la politique combinée donne lieu à une élévation du critère utilitariste, nous considérons que cette baisse est mise en oeuvre, et partons du nouvel équilibre stationnaire pour une dette réduite d'un point. En répétant cet exercice autant que nécessaire, nous déterminons le niveau de dette en-deçà duquel les agents ne sont plus prêts à accepter une telle politique, selon le critère utilitariste. Le Tableau 2 illustre ce résultat. Au regard de ce critère, il n'est jamais intéressant de réduire la dette en compensant par une baisse du transfert de 0,5 point à la date $t=0$ (colonne notée $7,7 \%$ ). Concernant une hausse transitoire du transfert de 0,5 point (soit un transfert transitoire de 8,7\%), la baisse de la dette accroît le bien-être jusqu'au seuil de 305\%. En compensant par un transfert transitoire de $9,2 \%$, la baisse de la dette se poursuit jusqu'au seuil $84 \%$. Pour le plus fort ajustement de transfert transitoire ici envisagé, soit 9,7\%, la déviation à la baisse de la dette cesse d'être bénéfique en $-28 \%$.

\begin{tabular}{lcccc}
\hline & \multicolumn{4}{c}{ Niveau de transfert } \\
\cline { 2 - 5 } Déviation de la dette & $7,7 \%$ & $8,7 \%$ & $9,2 \%$ & $9,7 \%$ \\
\hline $500 \rightarrow 499 \%$ & $-0,00720$ & 0,00260 & 0,00730 & 0,01189 \\
$400 \rightarrow 399 \%$ & & 0,00153 & 0,00681 & 0,01196 \\
$306 \rightarrow 305 \%$ & 0,00001 & & \\
$305 \rightarrow 306 \%$ & $-0,00001$ & & \\
$300 \rightarrow 299 \%$ & & 0,00576 & 0,01146 \\
$200 \rightarrow 199 \%$ & & 0,00389 & 0,01016 \\
$100 \rightarrow 99 \%$ & & 0,00071 & 0,00756 \\
$85 \rightarrow 84 \%$ & & 0,00001 & \\
$84 \rightarrow 83 \%$ & & $-0,00004$ & \\
$0 \rightarrow-1 \%$ & & & 0,00226 \\
$-27 \rightarrow-28 \%$ & & & & 0,00004 \\
$-28 \rightarrow-29 \%$ & & & & $-0,00005$ \\
\hline \hline
\end{tabular}

TABLE 2 - Gain/perte de bien-être en pourcentage de consommation pour une politique combinée de baisse permanente de la dette compensée par une variation transitoire du transfert.

Les résultats de ce choc combiné, où le gouvernement réduit la dette de $350 \%$ vers $349 \%$ du PIB, sont reportés sur les Graphiques 1 et 2 . Le Graphique 1 présente les trajectoires, lors la transition de l'état initial vers l'état final, du stock de capital, du taux d'intérêt, de l'offre de travail, de la consommation, du PIB et des taxes pour un ajustement à la date $t=0 \mathrm{du}$ transfert de $-0,5$ point (soit un transfert de $7,7 \%$ ) et $+0,5$ point (soit un transfert de 8,7\%). La baisse-comme la hausse- transitoire du transfert, 
couplée à une baisse permanente de la dette, s'accompagne d'une hausse immédiate du niveau des taxes. A l'issue de la transition, toutefois, le désendettement permet de réduire les taxes par rapport à leur niveau initial. La hausse des taxes à la date $t=0$ exerce un effet négatif sur l'offre de travail, mais à long terme, le désendettement marginal de l'Etat, en réduisant les prélèvements à long terme, a tendance à accroître la quantité de travail, par rapport à l'équilibre stationnaire initial. La consommation baisse immédiatement en raison de la hausse des taxes et de la baisse de l'offre de travail, mais se fixe à un niveau plus élevé dans l'état final que dans l'état initial. La hausse transitoire des taxes tend à abaisser l'épargne des ménages. A long terme, en revanche, l'effet d'éviction du capital privé par la dette publique est réduit, et le capital s'élève par rapport à son niveau initial. Les effets sur le taux d'intérêt brut et le PIB sont la résultante des ajustements de l'offre de travail et du stock de capital.
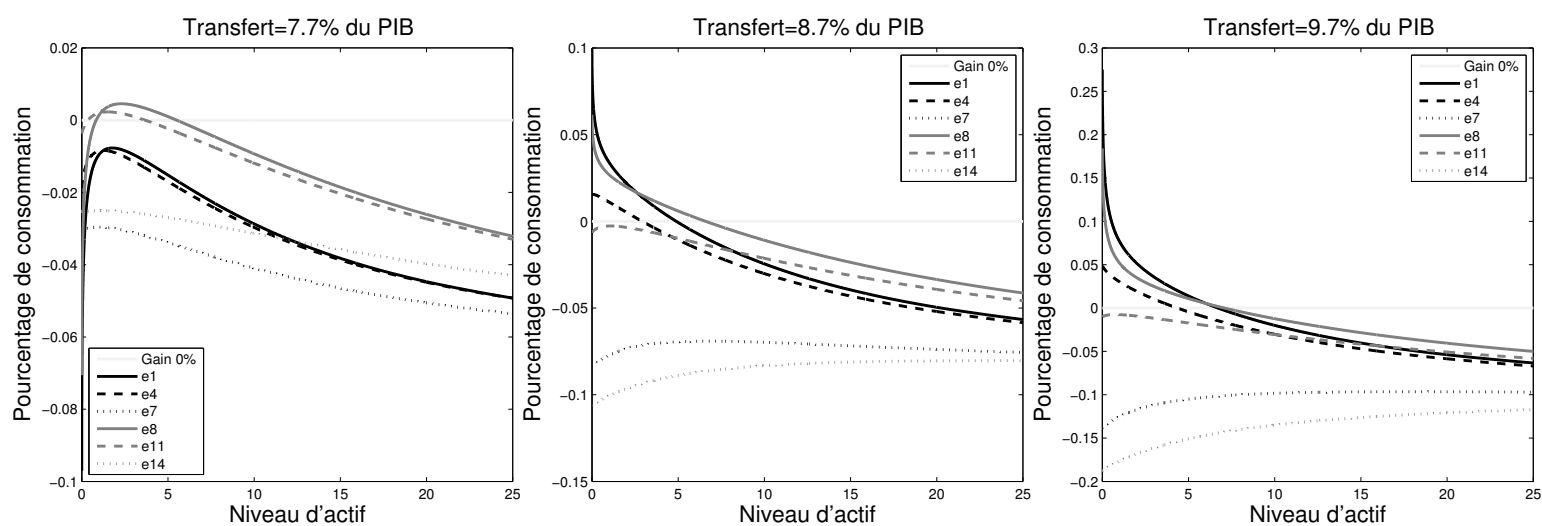

FIGURE 2 - Gain de bien-être pour une baisse de la dette de 350\% vers 349\%, pour différents niveaux de transfert et différentes catégories de productivité.

Sur le Graphique 2, nous considérons les effets d'un ajustement transitoire du transfert passant de 8,2\% du PIB aux différentes valeurs suivantes : 7,7\%, 8,7\% et 9, 7\% du PIB. Le graphique présente le gain de bien-être mesuré en points de consommation permanente que cette politique procure par rapport au status quo, et ceci pour certaines catégories de productivité ${ }^{6}$, en fonction de leur stock d'actifs financiers.

Dans le cas où l'ajustement transitoire du transfert est à 7,7\% -qui correspond ici à une baisse du transfert de 0,5 point- on observe (c.f. Graphique 1) une hausse du taux de taxe à court terme, qui est toutefois faible, car la baisse des transferts compense partiellement l'effort de désendettement. Cette hausse de la taxation est bien plus modérée dans ce cas que dans ceux où le transfert augmente à la date initiale.

6. Sur les 7 niveaux de productivité temporaire, nous représentons les niveaux extrêmes et le niveau médian. 
Seules quelques catégories particulières de productivité sont favorables à cette politique et ceci pour des niveaux courant d'actif bas. Il s'agit des catégories e8 et plus marginalement $e 11$, i.e. deux catégories ayant une composante permanente de productivité élevée et une composante transitoire de productivité faible ou modérée. Il s'agit des catégories d'agents les plus favorables à une hausse dans le long terme du salaire. En effet, la politique de baisse permanente de la dette s'accompagne d'une baisse du taux d'intérêt et d'une hausse du salaire à long terme. Pour ces agents, le gain à long terme de la hausse du salaire et de la baisse des taxes compense un choc transitoire de transfert négatif et une légère hausse du taux de prélèvement. Pour les agents appartenant à ces catégories de productivité et ne disposant pas d'actifs financiers, la baisse du transfert les affecte davantage, c'est pourquoi ils voient leur bien-être se détériorer. Pour les agents à salaire courant élevé (catégories $e 7$ et $e 14$ ), la hausse du taux de taxe est particulièrement coûteuse.

Dans le cas de la hausse transitoire du transfert, les effets sont similaires, que l'amplitude du choc s'élève à 0,5 point (transferts transitoires de $8,7 \%$ du PIB) ou à 1,5 point (transferts de 9,7\%). La hausse à court terme du taux de taxe est acceptée, car elle est plus que compensée par la hausse transitoire du transfert pour la plupart des catégories d'agents lorsque leur niveau d'actif courant est relativement faible. Lorsque la richesse financière des agents s'accroît, cette politique cesse d'être souhaitée, car d'une part cette hausse transitoire des taxes n'est pas la bienvenue et, d'autre part, à long terme cette politique s'accompagne d'une baisse du taux d'intérêt. Enfin, on peut noter dans cet exemple que pour certaines catégories de productivité, la politique de baisse de la dette accompagnée d'une hausse transitoire du transfert donne toujours lieu à une détérioration du bien-être. Il s'agit des agents dont la composante transitoire de productivité est la plus élevée. En effet, la ponction fiscale accrue vient réduire le salaire net précisément lorsque celui-ci est transitoirement élevé, et les effets favorables de long terme ne parviennent pas à compenser cette perte.

\subsection{Tentation de dévier en termes de dette et de transfert}

La section précédente a fourni une illustration de combinaisons de chocs sur la dette et les transferts rendant acceptable le désendettement de l'Etat. Bien que le caractère arbitraire $\mathrm{du}$ choix de ces combinaisons particulières puisse se comprendre et se défendre, la tentation de dévier à nouveau vers davantage de dette publique se fera mécaniquement sentir, car l'économie sera simplement revenue à une situation où la dette publique est réduite, et les transferts se situeront à leur niveau initial. 
Pour envisager des situations finales pour lesquelles la dette publique serait abaissée par rapport au ratio de $686 \%$ mis en exergue précédemment, et où la tentation d'élever à nouveau la dette aurait disparu, il est clair qu'un autre instrument fiscal devra être modifié. Le cadre épuré du modèle invite naturellement à considérer les transferts comme étant cet autre instrument. Pour éviter le caractère arbitraire d'un choc exogène sur les transferts, on peut alors se poser la question des tentations de dévier vers davantage de transferts, de manière symétrique à celle pour la dette publique.

La tentation de dévier vers plus -ou moins- de transferts peut être décrite à dette publique donnée, mais alors, pour ce nouveau niveau de transferts, on peut se demander dans quelle mesure les autorités pourraient souhaiter dévier en termes de dette publique. Cette dernière étant modifiée, la tentation de dévier en termes de transferts risque à son tour d'en être affectée, et ainsi de suite. Ce raisonnement circulaire invite à chercher un équilibre à partir duquel l'Etat n'aurait plus aucune tentation de dévier, ni en termes de dette, ni en termes de transferts. A cette fin, nous proposons de considérer les ajustements suivants : (1) en partant de l'équilibre stationnaire initial correspondant au modèle calibré ( $b=2 / 3$ et $\chi=8,2 \%$ ), nous simulons les effets d'un accroissement marginal (1 point) instantané et définitif du ratio dette/PIB jusqu'à trouver la situation au-delà de laquelle l'Etat ne souhaite plus dévier $(686 \%) ;(2)$ en considérant cet état final comme nouvel équilibre initial, nous testons les déviations instantanées et définitives en termes de transfert -à la hausse comme à la baisse- qui engendre des gains en bien-être. De proche en proche, nous ajustons le transfert, et simulons les transitions d'un état stationnaire à un autre, jusqu'à aboutir à une situation telle qu'aucune variation ultérieure ne soit désirable ; (3) à partir de cet état, nous revenons à l'étape précédente en simulant les effets de la déviation en termes de dette, et ainsi de suite.

Si ce processus converge, alors l'équilibre final est tel que le bien-être ne peut être accru ni par un ajustement permanent de la dette, ni par un ajustement permanent des transferts. Cet algorithme ne garantit toutefois pas, ni l'existence de pareil équilibre, ni son unicité ${ }^{7}$. Une condition nécessaire évidente à l'existence et à l'unicité de l'équilibre est que ce processus converge quel que soit l'équilibre stationnaire initial. Enfin, il est clair que cet équilibre est stable au sens de la déviation unilatérale de chacun des deux instruments fiscaux considérés, mais cela ne garantit pas qu'il n'existe aucune combinaison de chocs simultanés de ces deux instruments qui accroisse le bien-être

7. Comme il est usuel avec ce type de modèle résolu numériquement, la convergence est gage de l'existence. En revanche, l'unicité ne peut être déduite de simulations. 
social.

Pour illustrer ce processus de tâtonnement, nous partons de l'équilibre stationnaire correspondant au calibrage du modèle, et implémentons deux processus de déviations successives : le premier, respectant le cheminement décrit ci-dessus et le second, différant du premier par le fait que la première déviation porte sur les transferts et non sur la dette ${ }^{8}$. Sur le Graphique 3 nous représentons par des traits épais la succession de déviations pour des variations de la dette de \pm 1 point et des transferts de $\pm 0,05$ point.

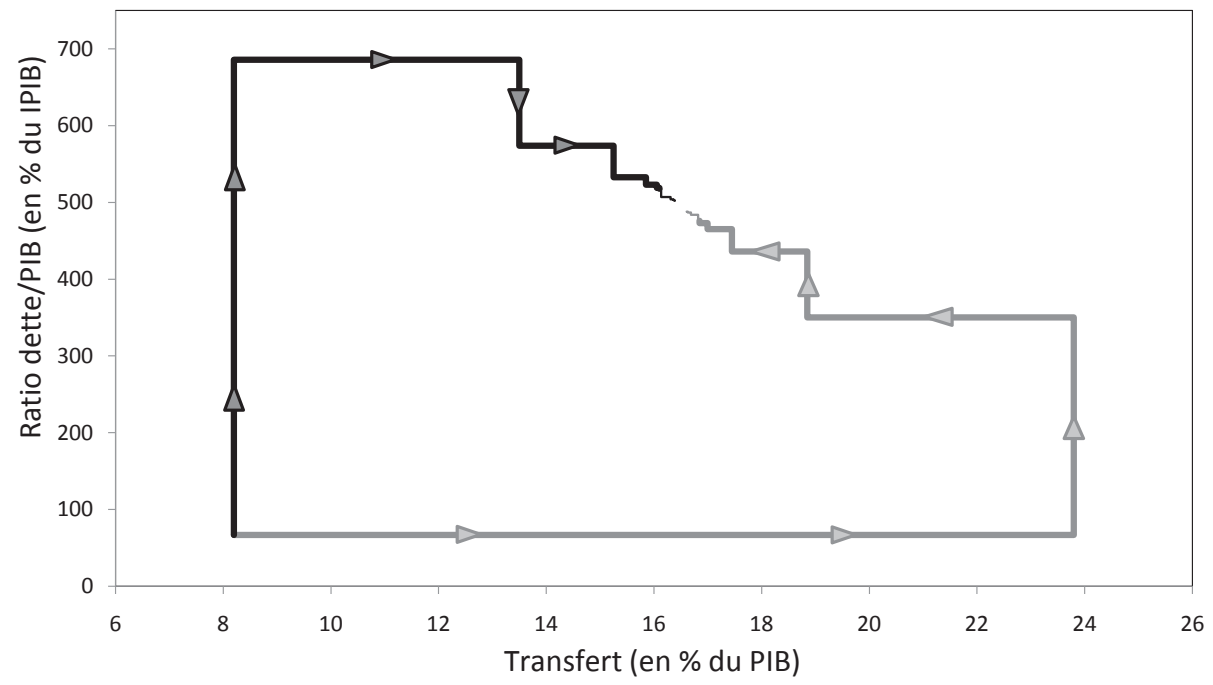

FIGURE 3 - Trajectoires de déviations successives en dette et transfert.

De ces graphiques, il ressort clairement que : (1) le transfert initial $(8,2 \%)$ est fortement sous-optimal, quel que soit le niveau de la dette publique ; (2) à chaque étape des deux cheminements, le transfert vers lequel l'Etat est tenté de dévier est d'autant plus élevé que la dette publique est basse ; (3) inversement, la dette publique optimale au sens des déviations est d'autant plus élevée que le transfert est bas.

On peut également noter que les deux simulations, si elles décrivent des couples (dette, transfert) qui se rapprochent progressivement l'un de l'autre, n'aboutissent pas exactement au même équilibre, le processus de convergence des deux trajectoires étant stoppé avant son achèvement. La trajectoire sur laquelle la dette croît atteint le point $(b=476 \% ; \chi=16,85 \%)$ et l'autre trajectoire atteint finalement $(b=518 \% ; \chi=16,10 \%)$. Ceci tient à ce que les effets sur le bien-être de chocs opposés et de même amplitude (dans ces simulations, \pm 1 point pour le ratio dette/PIB et $\pm 0,05$ point pour le transfert), ne sont pas exactement symétriques. Il s'ensuit que pour certains équilibres, le status quo est préférable à tout choc, à la hausse comme à la baisse. Cette préférence

8. L'état initial est donc identique, mais les transferts sont ajustés prioritairement. 
pour l'inaction est due à l'amplitude du choc considérée ici : si un choc très faible par exemple, un choc à la hausse de 0,1 point de la dette- est souhaité, il est possible qu'un choc de 1 point ne soit, lui, pas désiré (a fortiori, une baisse de 1 point ne sera pas désirée non plus). Pour tester cette conjecture, nous partons du dernier équilibre de chacune des deux trajectoires, et considérons désormais des chocs de 0,2 point de PIB pour la dette et de 0,01 point de PIB pour le transfert. Ces deux trajectoires apparaissent également sur le Graphique 3, par les trajectoires en trait fin, qui poursuivent celles en trait épais.

La prise en compte de chocs d'amplitude réduite permet de prolonger considérablement le processus d'ajustement. La trajectoire ascendante en termes de dette atteint le point ( $b=488 \% ; \chi=16,61 \%)$ et la trajectoire descendante en dette, le point $(b=502 \% ; \chi=16,39 \%)$. En mesurant l'écart de convergence entre les deux trajectoires par la différence des ratios dette/PIB finaux, on voit qu'en ayant divisé par un facteur 5 l'amplitude des chocs, l'écart est lui-même divisé par 3 ([488\%; 502\%] contre $[476 \% ; 518 \%]$ précédemment), et devient négligeable ${ }^{9}$. On peut donc considérer que l'équilibre stable au sens des déviations unilatérales en dette et en transfert, se situe aux environs de $b=495 \%$ et $\chi=16,5 \%$.

Rappelons que Floden (2001) a montré que l'optimum de long terme par rapport à la dette et au transfert est atteint pour un ratio dette/PIB de $-100 \%$ et un transfert de $23 \%$ du PIB. Son analyse, reposant sur des comparaisons d'états stationnaires, ne prend pas en compte les effets de court terme. Il apparaît donc qu'à l'optimum de long terme, la tentation de dévier vers davantage de dette -prise en compte dans la dynamique transitoire- est trop forte et qu'en conséquence il est souhaitable de réduire les transferts. L'effet de la tentation de dévier en termes de dette sur l'ajustement ultérieur du transfert est loin d'être négligeable, puisque ce dernier qui vaut $23 \%$ à l'optimum de long terme, ne vaut plus qu'environ 16,5\% ici. L'effet sur le ratio dette/PIB d'équilibre est lui encore plus fort, puisqu'à l'équilibre stationnaire, la dette publique maximisant le critère utilitariste vaut $-100 \%$, contre environ $495 \%$ ici.

Pour mieux comprendre pourquoi l'optimum de long terme relatif à la dette publique et aux transferts donne lieu à des tentations de dévier vers davantage de dette et des transferts réduits, nous pouvons reprendre le processus de déviations successives de la dette et des transferts présenté ci-dessus, en partant de l'optimum de long terme mis en avant par Floden (2001). Quel que soit l'ordre des déviations -débutant par un

9. En l'état, nous ne pouvons ni prouver, ni même illustrer numériquement, que la prise en compte de chocs d'amplitude arbitrairement faible garantirait la convergence totale des deux trajectoires, quoiqu'il soit raisonnable de le supposer. 
ajustement, soit de la dette, soit des transferts-, le transfert finit par converger vers approximativement $16,5 \%$ et la dette s'élève jusqu'aux environs de $495 \%$ (trajectoires non reproduites). Ce fort ajustement est dû au fait que, lorsque la dette publique est très basse -voire négative-, la tentation de créer davantage de dette est considérable. Ceci peut être illustré par l'effet de la hausse de 1 point du ratio dette/PIB en comparant deux situations initiales : l'optimum de long terme de Floden (2001) d'une part $(b=-100 \% ; \chi=23 \%)$, et d'autre part l'équilibre stationnaire caractérisé par $(b=100 \% ; \chi=23 \%)$. Le Tableau 3 présente les effets de ce choc sur le bien-être collectif, ainsi que sur la proportion d'agents favorable à cette politique.

\begin{tabular}{lcc}
\hline \hline & $b=-100 \% ; \chi=23 \% \%$ & $b=100 \% ; \chi=23 \% \%$ \\
$\frac{\Delta C}{C}$ & $+0,020 \%$ & $+0,005 \%$ \\
Prop. agents en faveur & $96,0 \%$ & $56,8 \%$ \\
\hline \hline
\end{tabular}

TABLE 3 - Effets sur le bien-être et proportions d'agents en faveur d'une hausse de 1 point du ratio dette/PIB.

Il est patent que les gains d'une hausse de la dette sont d'autant plus forts que la dette est très éloignée du niveau au-delà duquel les déviations ne sont plus souhaitables (qui, pour un transfert de $23 \%$, se situe à 363\%). Le surcroît d'effet distorsif à long terme -à la fois sur l'offre de travail et sur l'offre de capital- provenant d'un accroissement de 1 point du ratio dette/PIB est sensiblement plus faible pour une dette elle-même très faible. C'est ce que le Graphique 4 représente : en normalisant à 1 la consommation agrégée à la date initiale pour chacune des deux trajectoires, on voit clairement que le coût à long terme en consommation est, en termes relatifs, plus faible pour une dette initialement très basse, et que les gains transitoires sont eux, nettement plus forts.

\section{Conclusion}

Dans cet article, et à la suite de Desbonnet et Weitzenblum (2011), nous étendons le modèle de Floden (2001) en modélisant explicitement la dynamique transitoire entre différents états stationnaires caractérisés par différents niveaux de dette publique et de transfert. Dans un premier temps, nous illustrons dans quelle mesure, si la dette publique se trouvait initialement à un niveau élevé dû à la tentation de dévier mise en exergue par Desbonnet et Weitzenblum (2011), une combinaison judicieuse de réduction 


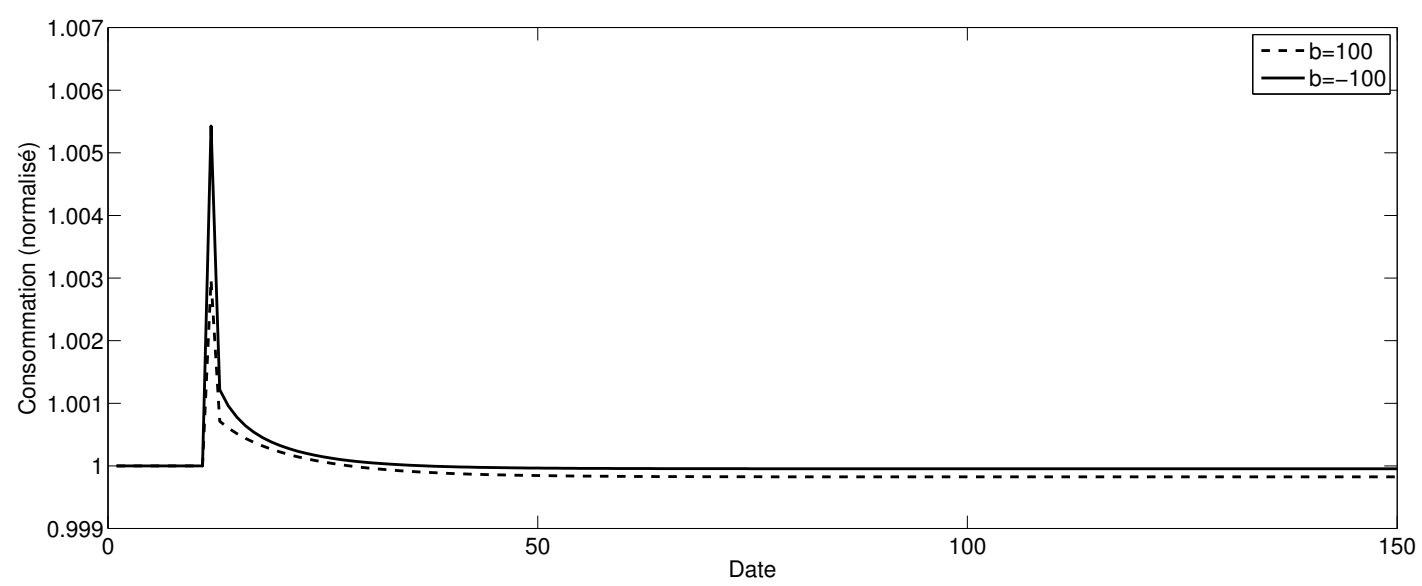

FIGURE 4 - Trajectoire de consommation normalisée.

de la dette et de hausse temporaire des transferts pourrait donner lieu à un ajustement qui ne détériorerait pas le bien-être collectif, tel que mesuré par le critère utilitariste. Dans un second temps, nous posons la question de la tentation de dévier à la fois en termes de dette et de transferts. Il apparaît que l'optimum en termes de dette et de transferts, proposé par Floden (2001) et reposant sur des comparaisons d'états stationnaires, n'est pas stable au sens qu'il donne lui-même lieu à une forte tentation de dévier. En partant d'une situation représentant de manière stylisée l'économie américaine, il apparait que les tentations de dévier cessent pour un transfert aux environs de 16,5\%, contrairement aux 23\% de Floden (2001). La dette publique, elle, se situe aux environs de $495 \%$, à comparer à l'excédent (-100\%) mis en avant par Floden (2001). Si l'ajustement du transfert donne lieu à de forts gains de long terme, les tentations de dévier vers davantage de dette en surprenant les agents demeurent substantielles, et illustrent une nouvelle fois à quel point la tentation de profiter de gains de courte période peut entrer en opposition avec l'objectif de bien-être de long terme. 


\section{Références}

AIYAGARI, S. (1994). Uninsured idiosyncratic risk and aggregate saving. The Quarterly Journal of Economics, 109(3):659-684.

Aiyagari, S., Marcet, A., SARgent, T. et Seppala, J. (2002). Optimal taxation without state-contingent debt. Journal of Political Economy, 110(6):1220-1254.

AiYAGARI, S. R. et MCGRATTAN, E. R. (1998). The optimum quantity of debt. Journal of Monetary Economics, 42(3):447-469.

BARRO, R. J. (1974). Are government bonds net wealth? The Journal of Political Economy, 82(6):1095-1117.

BARRO, R. J. (1979). On the determination of the public debt. The Journal of Political Economy, 87(5):940-971.

BEWLEY, T. (1986). Stationary monetary equilibrium with a continuum of independently fluctuating consumers. Contributions to Mathematical Economics in Honor of Gerard Debreu, 79.

DANIEL, B. (1993). Tax timing and liquidity constraints : A heterogeneous-agent model. Journal of Money, Credit and Banking, 25(2):176-196.

Desbonnet, A. et Weitzenblum, T. (2011). Why do governments end up with debt? short-run effects matter. Economic Inquiry, Forthcoming.

FLODEN, M. (2001). The effectiveness of government debt and transfers as insurance. Journal of Monetary Economics, 48(1):81 - 108.

FLODEN, M. et LINDE, J. (2001). Idiosyncratic risk in the united states and sweden : Is there a role for government insurance? Review of Economic Dynamics, 4(2):406-437.

HeAthсоте, J. (2005). Fiscal policy with heterogeneous agents and incomplete markets. Review of Economic Studies, 72(1):161-188.

HugGetT, M. (1993). The risk-free rate in heterogeneous-agent incomplete-insurance economies. Journal of Economic Dynamics and Control, 17(5-6):953 - 969.

LuCAS, R. E. et STOKEy, N. L. (1983). Optimal fiscal and monetary policy in an economy without capital. Journal of Monetary Economics, 12(1):55-93. 
TAUCHEN, G. (1986). Finite state markov-chain approximations to univariate and vector autoregressions. Economics letters, 20(2):177-181. 